\title{
K-CAPTURE COSMIC RAY SECONDARIES AND REACCELERATION
}

\author{
F.C. Jones ${ }^{1}$, A. Lukasiak ${ }^{2}$, V.S. Ptuskin ${ }^{2,3}$ and W.R. Webber ${ }^{4}$ \\ ${ }^{\prime}$ Laboratory for High Energy Astrophysics, NASA/GSFC, Greenbelt, MD 20771,USA. \\ ${ }^{2}$ Institute for Physical Science and Technology, University of Maryland, College Park, MD 20742,USA. \\ ${ }^{3}$ IZMIRAN, Russian Academy of Sciences, Troitsk, Moscow region 142092, Russia. \\ ${ }^{4}$ New Mexico State University, Las Cruses, NM 88003, USA.
}

\begin{abstract}
We have investigated the effect of reacceleration on interstellar flux of K-capture secondaries ${ }^{49} \mathrm{~V}$ and ${ }^{51} \mathrm{Cr}$. Several isotopic ratios for these two isotopes are calculated using the galactic diffusion model with and without distributed reacceleration. It is found that the statistical accuracy of the ACE experiment on itself is high enough to see a signature of reacceleration. However, the uncertainties in nuclear production cross sections are probably too large to conclude that reacceleration process took place.
\end{abstract}

\section{INTRODUCTION}

The isotopes ${ }^{37} \mathrm{Ar},{ }^{44} \mathrm{Ti},{ }^{49} \mathrm{~V},{ }^{51} \mathrm{Cr}$ and others are produced by nuclear interactions during cosmic ray propagation in the interstellar gas. They rapidly decay by electron capture at low energies when energetic ions can have an orbital electron. The probability to have a bound electron strongly depends on energy. As a consequence, the surviving fraction of $\mathrm{K}$-capture isotopes and the abundance of their decay products are strong functions of energy and are sensitive to the possible change of particle energy in the interstellar medium. This has led to suggestion (Silberberg et al.,1983; Silberberg and Tsao,1990; Soutoul et al., 1998 ) that the measurements of secondary K-capture isotopes in low-energy cosmic rays at $E<1$ $\mathrm{GeV} /$ nucleon could test the reacceleration hypothesis. The new ACE data (Niebur et al., 2000) on the isotopic composition of titanium, vanadium, and chromium have high statistical accuracy, show energy dependence of the content of radioactive electron capture isotopes, and can be used for the comprehensive investigation of interstellar propagation of low energy cosmic rays.

In this paper we study two K-capture processes ${ }^{5 !} \mathrm{Cr} \rightarrow{ }^{51} \mathrm{~V}$ and ${ }^{49} \mathrm{~V}->^{49} \mathrm{Ti}$. We have calculated several isotopic ratios involving $\mathrm{K}$-capture process using a disk-halo diffusion model with and without reacceleration (Jones et al., 2000). The effect of reacceleration is discussed in the context of uncertainties existing in measured isotopic ratios and nuclear fragmentation cross sections used in this study.

\section{PROPAGATION CALCULATIONS}

We have performed propagation calculations using the one-dimensional diffusion model with a thin disk of cosmic ray sources and matter, and with an extended matter-free halo $|z| \leq H$. Both models are described in detail in our paper Jones et al. (2000). The cosmic rays diffuse through the Galaxy with a momentum dependent diffusion coefficient $D$ and freely escape from the system at the halo boundaries $|z|=H$. It is assumed that diffusion results from the particle scattering on interstellar turbulence. In addition to the spatial diffusion, the energetic particles in the model with reacceleration experience stochastic acceleration which is described as diffusion on momentum with diffusion coefficient $D_{p p} \sim$ 
$p^{2} V_{\mathrm{a}}^{2} / D$, where $p$ is the particle momentum and $V_{\mathrm{a}}$ is the Alfven velocity. The reacceleration occurs in the region $|z| \leq h_{a}$. Following Seo and Ptuskin (1994), we take $h_{a}=H / 3$.

The escape length $X_{e}, g / \mathrm{cm}^{2}$, determines the mean matter thickness traversed by energetic particles observed in the Galactic disk without effects of fragmentation, decay, and energy change taken into account. The escape length is expressed through the parameters of the diffusion model as $X_{e}=\mu \beta c H /(2 D)$, where $\mu\left(\approx 2.4 \cdot 10^{-3} \mathrm{~g} / \mathrm{cm}^{2}\right)$ is the surface gas density of the Galactic gas disk, $\beta c$ is the particle velocity. It was found (Jones et al. 2000, see also Figure 1) that the following sets of parameters give the best fit to the data on cosmic ray secondary Boron and sub-Iron $(\mathrm{Sc}+\mathrm{Ti}+\mathrm{V})$ elements in two models under the consideration ( $R$ is the particle magnetic rigidity): $X_{e}=11.8 \beta \mathrm{g} / \mathrm{cm}^{2}$ at $R<4.9$ $\mathrm{GV}, X_{e}=11.8 \beta(R / 4.9 G V)^{-0.54} \mathrm{~g} / \mathrm{cm}^{2}$ at $R \geq 4.9 \mathrm{GV}$ in the model without reacceleration; and $X_{e}=9.4 \cdot R^{-0.3} \mathrm{~g} / \mathrm{cm}^{2}$ at all $R, V_{a}=40 \mathrm{~km} / \mathrm{s}$ in the model with reacceleration (the value of $V_{a}$ depends on the size of the reacceleration region as $V_{a} \propto h_{a}^{-1 / 2}$ ). It is worth noting that the spatial diffusion coefficient in the reacceleration model has a more "natural" scaling on rigidity, $D \propto \beta R^{0.3}$, that corresponds to the

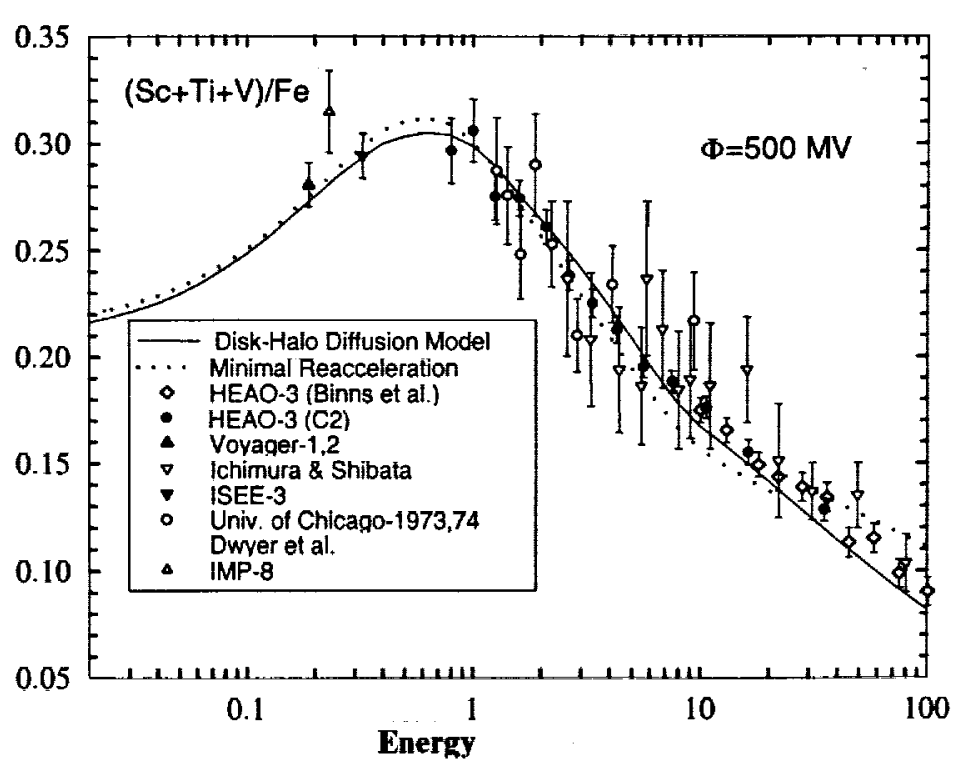

Figure 1. The least squares fit to observed sub $\mathrm{Fe} / \mathrm{Fe}$ ratios to two propagation models with (dotted line) and without (solid line) interstellar reacceleration. $F$ is the force field approximation solar modulation parameter. For references to data points see Jones et al., 2001. cosmic ray scattering on a Kolmogorovtype spectrum of interstellar turbulence. Stochastic reacceleration essentially modifies the spectra of primaries and secondaries below $10 \mathrm{GeV} / \mathrm{n}$ and reproduces the observed peaks in secondary/primary ratios at about $1 \mathrm{GeV} / \mathrm{n}$.

The nuclear cross sections used in our calculations include the new primary cross sections in hydrogen targets for $\mathrm{C}$ through $\mathrm{Ni}$ at $600 \mathrm{MeV} / \mathrm{n}$ as described in Webber, et al. (1998ab), as well as the hydrogen cross sections for essentially all of the secondary nuclei from $\mathrm{Li}$ through $\mathrm{Mn}$ also at $600 \mathrm{MeV} / \mathrm{n}$ reported in Webber et al. (1998c). The energy dependence of these isotopic cross sections is updated and extended as well using earlier charge changing cross sections measured between 300 and $1700 \mathrm{MeV} / \mathrm{n}$ (Webber et al. 1990) and at $15 \mathrm{GeV} / \mathrm{n}$ (Webber et al. 1994) and assuming that the isotopic fractions are generally energy independent as confirmed

by these earlier measurements and those of the Transport Collaboration (Chen et al. 1997). The atomic cross sections for attachment of a free electron are calculated as in Letaw et al. (1984).

\section{RESULTS, DISCUSSION AND CONCLUSION}

The results of propagation calculations for two isotopic ratios ${ }^{49} \mathrm{Ti} /{ }^{49} \mathrm{~V}$ and ${ }^{51} \mathrm{Cr} /{ }^{51} \mathrm{~V}$ are displayed in Figure 2. The solid curve represents the model without reacceleration and the dotted line corresponds to the reacceleration model. The experimental points are from new high resolution and high statistics data from the Cosmic Ray Isotope Spectrometer on the ACE spacecraft (Niebur et al., 2000) and from the Ulysses experiment (Connell and Simpson, 1999). Cosmic ray isotopes ${ }^{51} \mathrm{Cr}$ and ${ }^{49} \mathrm{~V}$ are produced by fragmentation of heavier cosmic ray nuclides and decay only by electron capture. The calculated isotopic ratio ${ }^{51} \mathrm{~V} /{ }^{51} \mathrm{Cr}$ in both models shows a strong dependence on energy. In the low energy region 100-300 


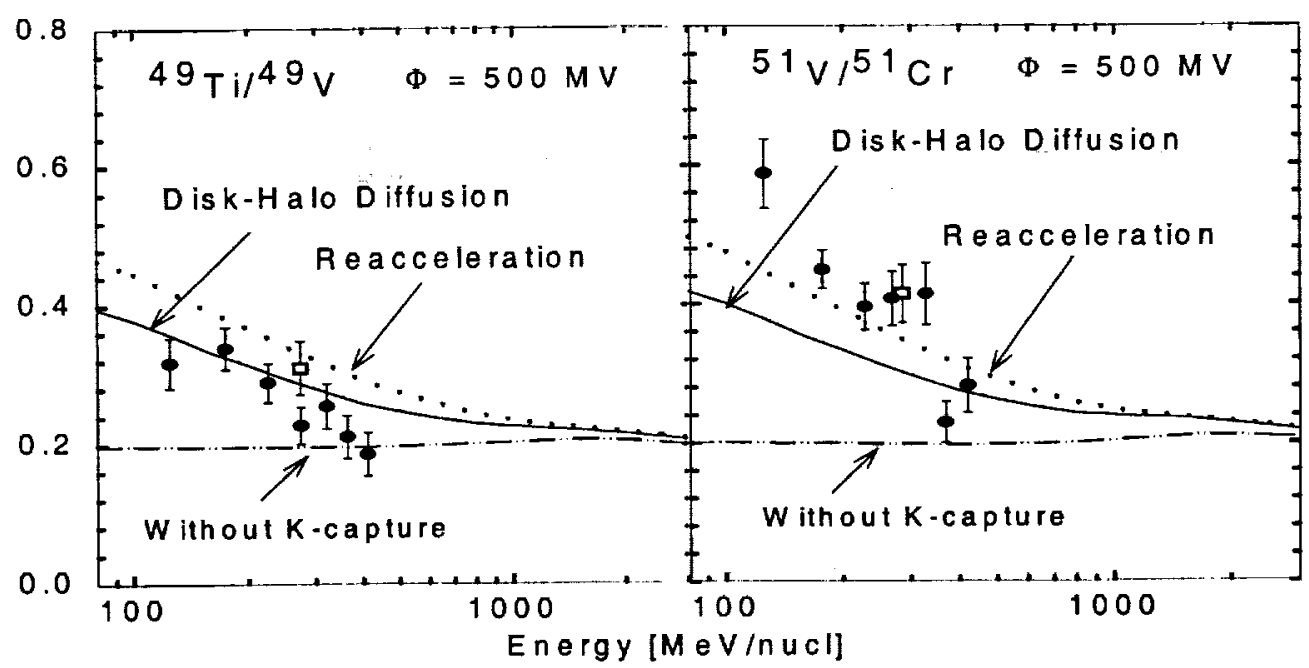

Figure 2 The isotopic ratios $49 \mathrm{Ti} / 49 \mathrm{~V}$ and $51 \mathrm{~V} / 51 \mathrm{Cr}$ as a function of the interplanetary kinetic energy. Full circles: CRIS/ACE (Niebur et al., 2000), open square: Ulysses (Connell and Simpson, 1999). The results from propagation calculations with K-capture dependence on energy. The dash-dotted curve corresponds to calculations

$\mathrm{MeV} / \mathrm{n}$ the effect of $\mathrm{K}$-capture process is strong and the isotopic ratio is twice larger than a ratio calculated without $\mathrm{K}$-capture process. The ${ }^{51} \mathrm{~V} /{ }^{51} \mathrm{Cr}$ isotopic ratio calculated in the model with reacceleration fits measured experimental points better than the model without reacceleration.

${ }^{51} \mathbf{V}^{F^{1}} \mathrm{Cr}$ - this isotopic ratio (Fig. 2) calculated in the reacceleration model at the energy of $200 \mathrm{MeV} / \mathrm{n}$ and modulation level of $500 \mathrm{MV}$ is larger by $17 \%$ than the ratio obtained in the model without reacceleration. This difference is slightly larger than 1 sigma uncertainty in the ACE measurement. In Fig 2 the results for ${ }^{51} \mathrm{~V} /{ }^{51} \mathrm{Cr}$ from the reacceleration model (dotted line) are in better agreement with measured

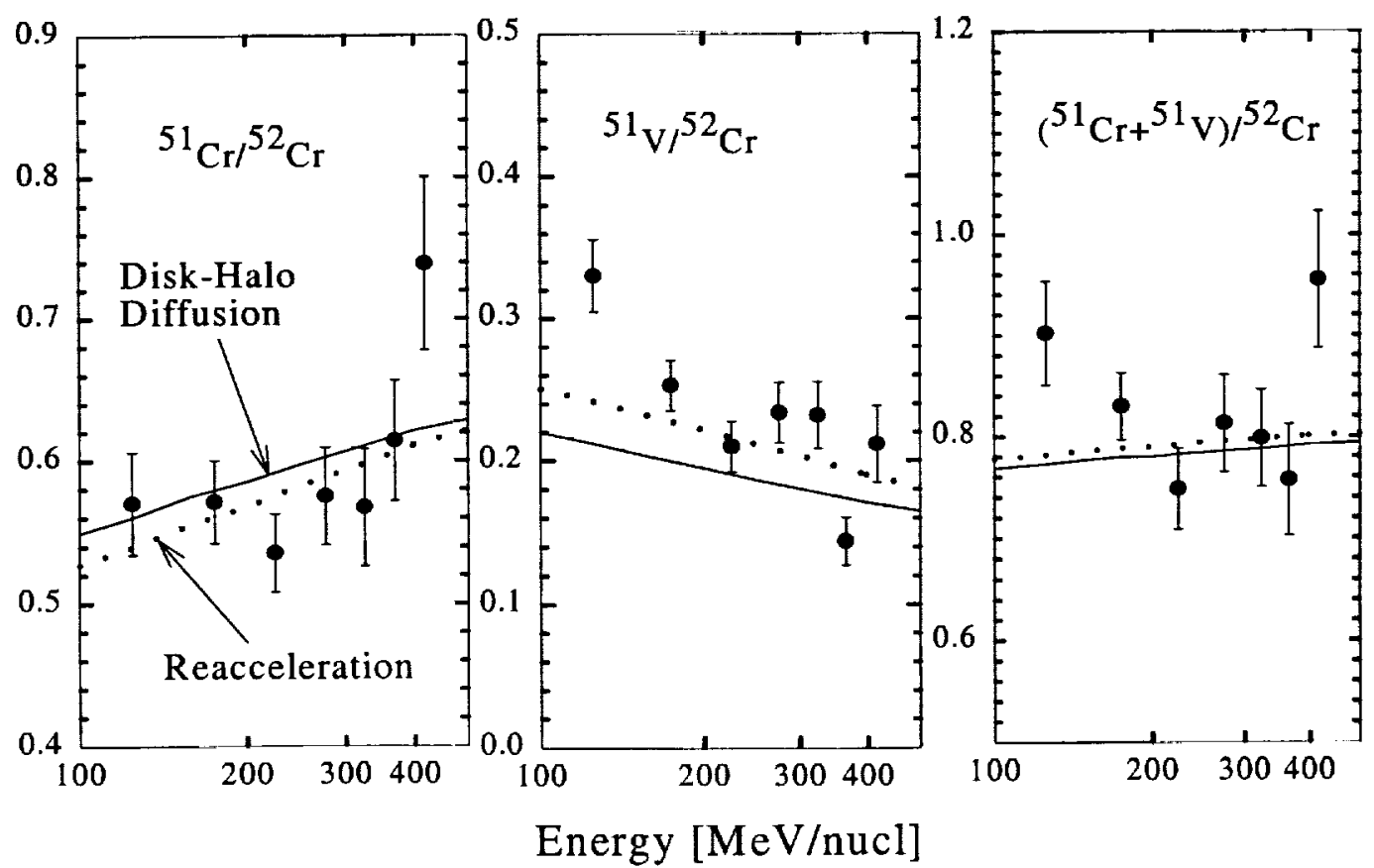

Figure 3 The isotopic ratios of parent and daughter abundances for the $51 \mathrm{Cr}->51 \mathrm{~V}$ decay. Solid curves represent the model without reacceleration and, dotted curves represent the model with reacceleration. 
isotopic ratios than the model without reacceleration (solid line).

$\left({ }^{51} \mathbf{C r}+{ }^{51} \mathbf{V}\right) /^{52} \mathrm{Cr}$ - in Figure 3 we present the isotopic ratio $\left({ }^{51} \mathrm{Cr}+{ }^{51} \mathrm{~V}\right) /{ }^{52} \mathrm{Cr}$ which involves parent and daughter and is constant with energy and independent on $\mathrm{K}$-capture process. The propagation calculations from both models are in good agreement with measured isotopic ratio from ACE experiment. This would indicate that used cross sections for fragmentation to ${ }^{51} \mathrm{Cr},{ }^{52} \mathrm{Cr}$ and ${ }^{51} \mathrm{~V}$ are rather well measured.

${ }^{51} \mathrm{~V}^{\boldsymbol{F}^{2}} \mathbf{C r}$ - the results from propagation calculations (Fig 3) indicate that the reacceleration model is in better agreement with measured ratios.

${ }^{51} \mathrm{Cr} /{ }^{52} \mathrm{Cr}$ - for this isotopic ratio (Fig. 3) results from the reacceleration model agree with ACE experiment only slightly better than the model without reacceleration. The difference between both models for this isotopic ratio is smaller than one sigma uncertainty in ACE measurement.

${ }^{49} \mathrm{Ti}^{49} \mathrm{~V}$ - this isotopic ratio calculated in both models is slightly higher than the measured ACE ratio. This could indicate that $\mathrm{K}$-capture cross sections are too large or that the fragmentation cross sections to ${ }^{49} \mathrm{Ti}$ are too large or that the ones to ${ }^{49} \mathrm{~V}$ are too small. The ${ }^{49} \mathrm{Ti}$ isotope is almost completely secondary with negligible source abundance of ${ }^{49} \mathrm{Ti} /{ }^{56} \mathrm{Fe}=0.02 \%$. Most of fragmentation $(\approx 80 \%)$ to ${ }^{49} \mathrm{Ti}$ comes from 5 isotopes with the largest contribution from ${ }^{56} \mathrm{Fe}\left({ }^{56} \mathrm{Fe} \rightarrow{ }^{49} \mathrm{Ti}=53.5 \%,{ }^{50} \mathrm{~V} \rightarrow{ }^{49} \mathrm{Ti}=8.7 \%,{ }^{52} \mathrm{Cr}-\right.$ $>{ }^{49} \mathrm{Ti}=8.6 \%,{ }^{51} \mathrm{Cr}->{ }^{49} \mathrm{Ti}=5.2 \%$ and ${ }^{50} \mathrm{Ti}->{ }^{49} \mathrm{Ti}=3.9 \%$ ).

The ${ }^{49} \mathrm{~V}$ is a pure secondary isotope with $\mathrm{K}$-capture process. The $80 \%$ of fragmentation to ${ }^{49} \mathrm{~V}$ comes from 7 contributions $\left({ }^{56} \mathrm{Fe}->{ }^{49} \mathrm{~V}=50.8 \%,{ }^{50} \mathrm{~V}->^{49} \mathrm{~V}=7.0 \%,{ }^{52} \mathrm{Cr}->{ }^{49} \mathrm{~V}=4.6 \%,{ }^{51} \mathrm{Cr}->{ }^{49} \mathrm{~V}=4.5 \%,{ }^{50} \mathrm{Cr}-\right.$ $>{ }^{49} \mathrm{~V}=4.3,{ }^{54} \mathrm{Fe}-{ }^{49} \mathrm{~V}=4.6 \%$ and ${ }^{57} \mathrm{Fe}->{ }^{49} \mathrm{~V}=4.3 \%$ ).

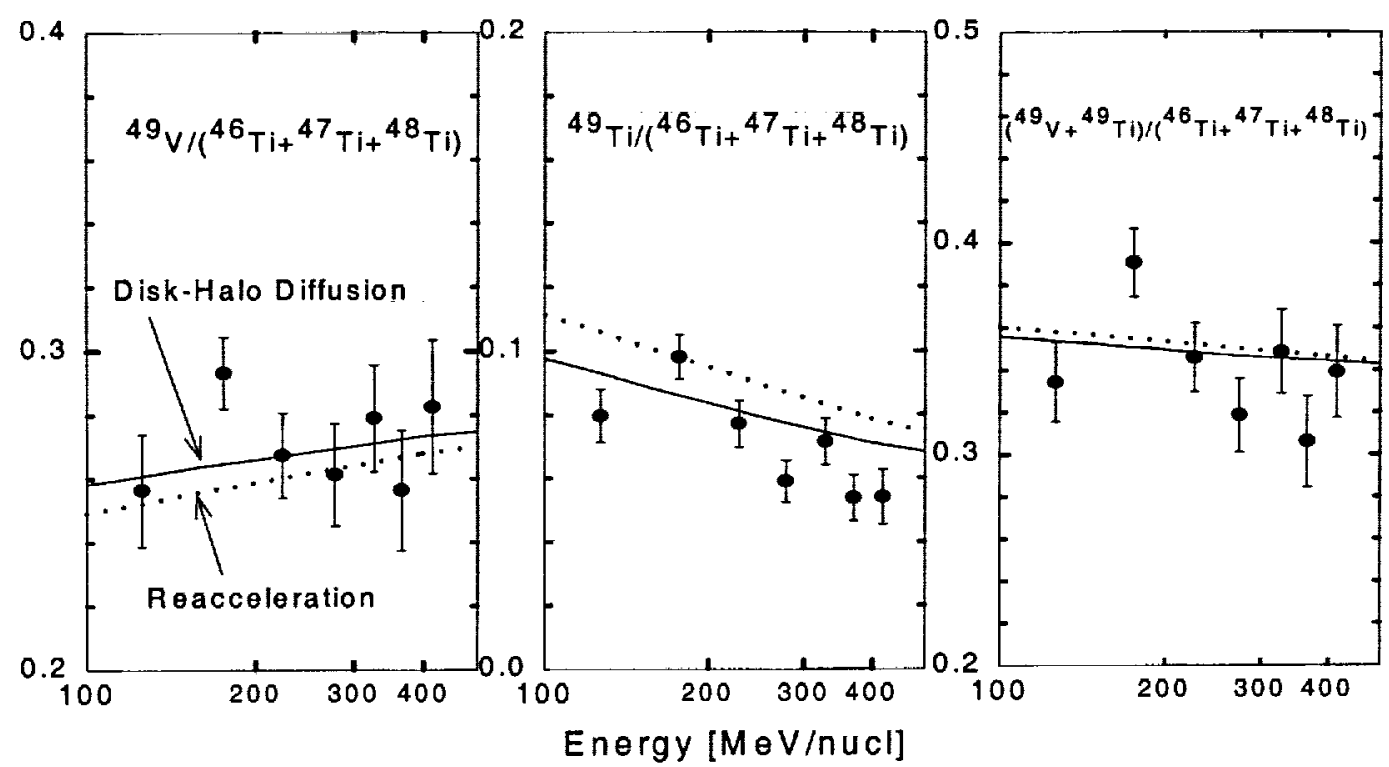

Figure 4 The isotopic ratios of parent and daughter isotopic ratios for the $49 \mathrm{~V}->49 \mathrm{Ti}$ decay. Solid curves represent the model without reacceleration and, dotted curves represent the model with reacceleration 
$\left({ }^{49} \mathrm{~V}+{ }^{49} \mathrm{Ti}\right) /\left({ }^{46} \mathrm{Ti}+{ }^{47} \mathrm{Ti}+{ }^{48} \mathrm{Ti}\right)$ - This combined isotopic ratio (Fig. 4) is used as a consistency test for propagation calculations. There is no K-capture effect in this ratio and it is almost constant with energy. The propagation calculations are higher than the average value from all 7 experimental points. This would indicate that fragmentation cross sections to ${ }^{49} \mathrm{Ti}$ are too large. See also in the same figure $\left.{ }^{49} \mathrm{Ti} /{ }^{46} \mathrm{Ti}+{ }^{47} \mathrm{Ti}+{ }^{48} \mathrm{Ti}\right)$.

${ }^{49} \mathrm{Ti} /\left({ }^{46} \mathrm{Ti}+{ }^{47} \mathrm{Ti}+{ }^{48} \mathrm{Ti}\right)$ - the propagation results (Fig. 4) from both models are higher than the experimental points. This indicates that fragmentation cross sections to ${ }^{49} \mathrm{Ti}$ are too large.

${ }^{49} \mathrm{~V} /\left({ }^{46} \mathrm{Ti}+{ }^{47} \mathrm{Ti}+{ }^{48} \mathrm{Ti}\right)$ - both models are in agreement with experimental points. The difference between both models is small when compared to 1 sigma uncertainty in measured values.

Reduction in the production cross sections to ${ }^{49} \mathrm{Ti}$ by $20 \%$ (twice more than is the uncertainty in the measured cross sections to ${ }^{49} \mathrm{Ti}$ ) improves the agreement of both models with measured $\left({ }^{49} \mathrm{~V}+{ }^{49} \mathrm{Ti}\right) /\left({ }^{46} \mathrm{Ti}+{ }^{47} \mathrm{Ti}+{ }^{48} \mathrm{Ti}\right),{ }^{49} \mathrm{Ti} /\left({ }^{46} \mathrm{Ti}+{ }^{47} \mathrm{Ti}+{ }^{48} \mathrm{Ti}\right)$ and ${ }^{49} \mathrm{Ti} /{ }^{49} \mathrm{~V}$ isotopic ratios. Such a reduction would give some advantage to the model with reacceleration. However, the existing uncertainties in cosmic ray data and, in particular, in the data on nuclear cross sections do not allow us to conclude that interstellar reacceleration process takes place. To fully understand the uncertainty of these conclusions one should perform a sensitivity analysis of the cross sections and propagation parameters. We have not done this for this presentation. This will be done in a later work.

\section{REFERENCES}

Chen, C.-X., Albergo, S., Caccia, Z., Costa, S., Crawford, H. J. et al., Relativistic Interaction of $22 \mathrm{Ne}$ and $26 \mathrm{Mg}$ in Hydrogen and the Cosmic-Ray Implications, ApJ, 479, 504, 1997

Connell, J.J., and Simpson, J.A., Ulysses HET measurements of electron-capture secondary isotopes: test the role of cosmic ray reacceleration, $26^{\text {th }}$ Intern. Cosmic Ray Conf., Salt Lake City, 3, 33-36, 1999.

Jones, F.C., Lukasiak, A., Ptuskin, V. and Webber, W., The Modified Weighted Slab Technique: Models and Results, $A p J, 547,264,2001$.

Letaw, J.R., Silberberg, R. and Tsao, C.H., Propagation of heavy cosmic-ray nuclei, ApJ. Suppl., 56, 369, 1984.

Niebur, S.M., Binns, W. R., Christian, E. R., Cummings, A. C., George, J. S. et al., Secondary ElectronCapture Decay Isotopes and Implications for the Propagation of Galactic Cosmic Rays, Proceedings of the ACE 2000 Workshop (Indian Wells, 2000), AIP Conference Proceedings, 528, 406, 2000.

Seo, E.S. and Ptuskin, V.S., Stochastic reacceleration of cosmic rays in the interstellar medium, ApJ, 431, 705, 1994.

Silberberg, R., Tsao, C. H., Letaw, J. R. and Shapiro, M. M.., Distributed acceleration of cosmic rays, Phys. Rev. Lett., 51, 1217-1222, 1983.

Silberberg, R. and Tsao, C.H., Phys. Reports, 191 (6), 351, 1990.

Soutoul, A., Legrain, R., Lukasiak, A., McDonald, F. B., Webber, W. R.. et al., Evidence from Voyager and ISEE-3 spacecraft. Data for the decay of secondary K-electron capture isotopes during the propagation of cosmic rays in the Galaxy, A\&A, 336, L61-L64, 1998.

Webber, W.R, Kish, J.C., \& Schrier, D.A., Individual isotopic fragmentation cross sections of relativistic nuclei in hydrogen, helium, and carbon targets, Phys. Rev. C, 41, 547, 1990.

Webber, W. R., Binns, W. R., Crary, D. \& Westphall, M. New measurements of charge-changing cross sections in carbon and hydrogen targets above $2 \mathrm{GeV}$ per nucleon: Evidence for an energy dependence that may strongly affect estimates of the energy dependence of cosmic-ray diffusion in the galaxy, ApJ, 429, $764,1994$.

Webber, W. R., Kish, J. C., Rockstroh, J. M., Cassagnou, Y., Legrain, R. et al., Production cross sections of fragmentation from beams of $400-650 \mathrm{MeV}$ per nucleon ${ }^{9} \mathrm{Be},{ }^{1} \mathrm{~B},{ }^{12} \mathrm{C},{ }^{14} \mathrm{~N},{ }^{15} \mathrm{~N},{ }^{16} \mathrm{O},{ }^{20} \mathrm{Ne},{ }^{22} \mathrm{Ne},{ }^{56} \mathrm{Fe}$, and ${ }^{58} \mathrm{Ni}$ nuclei interacting in a liquid hydrogen target. I. Charge changing and total cross sections, $A p J$, 508, 940, 1998a.

Webber, W. R., Kish, J. C., Rockstroh, J. M., Cassagnou, Y., Legrain, R. et al., Production cross sections of fragmentation from beams of $400-650 \mathrm{MeV}$ per nucleon ${ }^{9} \mathrm{Be},{ }^{11} \mathrm{~B},{ }^{12} \mathrm{C},{ }^{14} \mathrm{~N},{ }^{15} \mathrm{~N},{ }^{16} \mathrm{O},{ }^{20} \mathrm{Ne},{ }^{22} \mathrm{Ne},{ }^{56} \mathrm{Fe}$, 
and ${ }^{58} \mathrm{Ni}$ nuclei interacting in a liquid hydrogen target. II. Isotopic cross sections of fragments, $A p J, \mathbf{5 0 8}$, 949,1998 b.

Webber, W. R., Soutoul, A., Kish, J. C., Rockstroh, J. M., Cassagnou, Y., et al., Measurement of charge changing and isotopic cross sections at $\sim 600 \mathrm{MeV}$ /nucleon from the interactions of $\sim 30$ separate beams of relativistic nuclei from ${ }^{10} \mathrm{~B}$ to ${ }^{55} \mathrm{Mn}$ in a liquid hydrogen target, Phys.Rev. C, 58, 3539, 1998c. 